\title{
Timing optimization utilizing order statistics and multichannel digital silicon photomultipliers
}

\author{
Shingo Mandai, ${ }^{*}$ Esteban Venialgo, and Edoardo Charbon \\ Faculty of Electrical Engineering, TU Delft Mekelweg 4, 2628 CD Delft, The Netherlands \\ *Corresponding author: s.mandai@tudelft.nl
}

Received October 22, 2013; revised December 20, 2013; accepted December 20, 2013; posted December 23, 2013 (Doc. ID 199868); published January 24, 2014

\begin{abstract}
We present an optimization technique utilizing order statistics with a multichannel digital silicon photomultiplier (MD-SiPM) for timing measurements. Accurate timing measurements are required by 3D rangefinding and time-offlight positron emission tomography, to name a few applications. We have demonstrated the ability of the MD-SiPM to detect multiple photons, and we verified the advantage of detecting multiple photons assuming incoming photons follow a Gaussian distribution. We have also shown the advantage of utilizing multiple timestamps for estimating time-of-arrivals more accurately. This estimation technique can be widely available in various applications, which have a certain probability density function of incoming photons, such as a scintillator or a laser source. (C) 2014 Optical Society of America

OCIS codes: (000.5490) Probability theory, stochastic processes, and statistics; (030.5290) Photon statistics; (040.5250) Photomultipliers; (120.3890) Medical optics instrumentation; (170.6920) Time-resolved imaging; (280.3400) Laser range finder.

http://dx.doi.org/10.1364/OL.39.000552
\end{abstract}

Photomultiplier tubes (PMTs) are widely used for cancer diagnostics tools, such as positron emission tomography (PET), scientific research and industrial instrumentation, spectrophotometry or fluorescence-lifetime imaging microscopy and in high energy physics.

A silicon photomultiplier ( $\mathrm{SiPM}$ ) is an alternative to PMTs; it is often preferred because of its tolerance to magnetic fields, compactness, and low-bias voltage. At least two flavors exist of SiPMs: analog and digital. An analog SiPM (A-SiPM) consists of an array of avalanche photodiodes operating in Geiger mode (also known as single-photon avalanche diodes or SPADs), whose avalanche currents are summed in one node [ $\underline{1}-\underline{6}]$. The resulting current is proportional to the number of detected photons, thus providing single- and multiplephoton detection capability. In a digital SiPM (D-SiPM), each SPAD voltage output is digitally added to generate digital signals for each photon detection. A SPAD may be turned off when its activity (dark count rate, DCR) is deemed too high (this SPAD is known as screamer [7]). In most D-SiPMs, the global output is directly routed to a time-to-digital converter (TDC) that is implemented on the same die to reduce external components and temporal noise. However, for both A-SiPM an D-SiPM, the number of acquired timestamps (recoded time) is one per event, conventionally.

In recent years, new D-SiPMs have been introduced with the capability of acquiring multiple timestamps [ $\underline{8}-11]$. Such devices are known as multichannel D-SiPMs or MD-SiPMs. Acquisition of multiple timestamps gives accurate timing resolution and tolerance from detector noise [11-14]. To date, the MD-SiPM with the highest density of TDC per SiPM (48TDCs/780 $\mu \mathrm{m} \times 800 \mu \mathrm{m})$ has been proposed by us in $[10,11]$. This feature makes it possible to acquire multiple timestamps without sharing a TDC for the first several photons (less than 10 photons) and to utilize them for estimating timing information of complex multiphoton events, such as scintillations. In case of a PET application, the first 5-10 photons are the most important, and the timestamps after those photons are generally not interesting. In SPAD-based $3 \mathrm{D}$ rangefinding applications, the detector operates in photon-starved mode, so it will not receive more than 10 photons, typically. However, it is well-known that the timing information of the detection can be distorted by the variation of the number of detected photons [15].

In this Letter, we present experimental results where multiple photons are detected with the MD-SiPM, and we also show how to optimize the timing estimation of events utilizing multiple timestamps assuming a 3D rangefinding application.

Figure 1(a) shows our measurement setup. The photons from the laser impinge on the detector with a given timing uncertainty. The probability density function (p.d.f.) describing this uncertainty follows a Gaussian distribution with $374 \mathrm{ps}$ FWHM. The laser light is attenuated by a neutral density filter (NDF), diffused by a diffuser, and projected to the MD-SiPM chip that has been designed based on [11]. The laser p.d.f. is constant for various photon levels, so the laser p.d.f. was characterized while operating in photon-starved mode (less than 0.001 photons per pulse). This p.d.f., $f(t)$, and its cumulative density function, $F(t)$, are used to calculate the $n$th order statistics corresponding to $n$th photon's p.d.f., $f_{k: n}(t)$, as below,

$$
f_{k: n}(t)=n\left(\begin{array}{c}
n-1 \\
k-1
\end{array}\right) f(t) F(t)^{k-1}(1-F(t))^{n-k}
$$

Figure 1(b) shows the MD-SiPM architecture; it consists of an array of $16 \times 26$ photo-detecting cells (called pixels hereafter), and a 48-fold column-parallel TDC. The size of the pixel array is $780 \mu \mathrm{m} \times 800 \mu \mathrm{m}$. The pixel size is $30 \mu \mathrm{m} \times 50 \mu \mathrm{m}$ with a $57 \%$ fill factor where a photon can be detected. In one column, three TDCs are implemented (to reduce the number of pixels per TDC) in an interlaced configuration. The pixels with DCR exceeding a threshold are disabled, so as to minimize spurious TDC activation. 


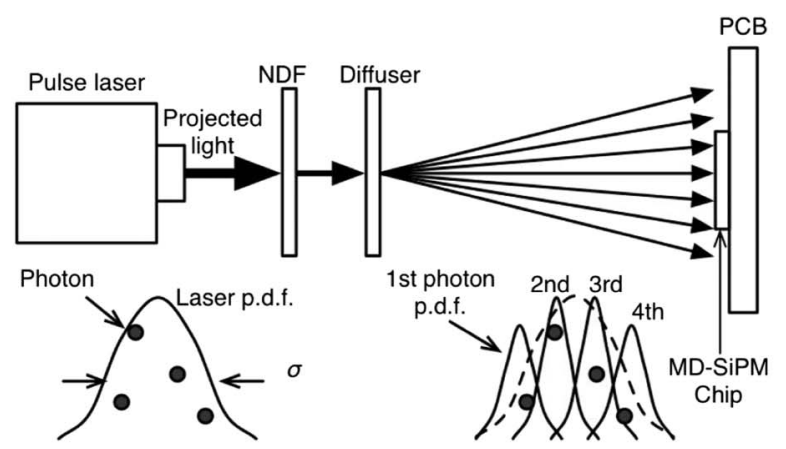

(a)

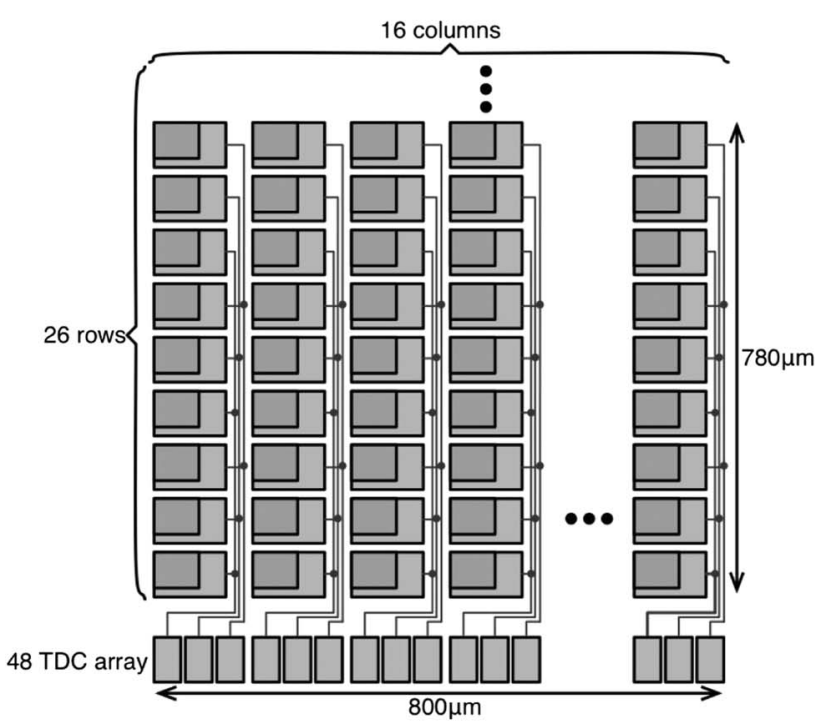

(b)

Fig. 1. (a) Measurement setup. (b) MD-SiPM structure.

Figure 2 shows each photon's calculated and measured p.d.f. when the laser intensity is $1,2,4$, and 9 photon level per MD-SiPM. The results prove that the MD-SiPM can acquire each photon's p.d.f. following the theory precisely. In practice, the optimized timing information of the TOA of a photon bunch is not necessarily obtained from the first photon, just as suggested by the theory. This is a typical feature of the photon bunch, when its p.d.f. is Gaussian, as in a laser, or a double-exponential, as in a conventional scintillator $[\underline{12}, \underline{13}, \underline{16}]$. Figure $\underline{3}$ shows the measurement results of each photon's timing resolution when the number of photons per pulse is 2,4 , and 9 per MD-SiPM. The rank of the best timing performance is second, third, and seventh at 2, 4 , and 9 photon level laser intensity, respectively, and their improvements are at most $37 \%$ in comparison to the first photon timing resolution. An MD-SiPM can acquire multiple photons so as to choose the best rank flexibly according to the detected number of photons. Figure $\underline{4}$ shows the summary of the timing resolution for each laser intensity. The timing resolution is improved by increasing the laser intensity until it is limited by intrinsic jitter, including the TDC jitter. The timing resolution is improved by the square root of the photon level, $N$, when each photon is independent and identically distributed (i.i.d) and follows a Gaussian distribution. Thus, by fitting an ideal curve, $303 / \sqrt{N}+70$, with the measurement,
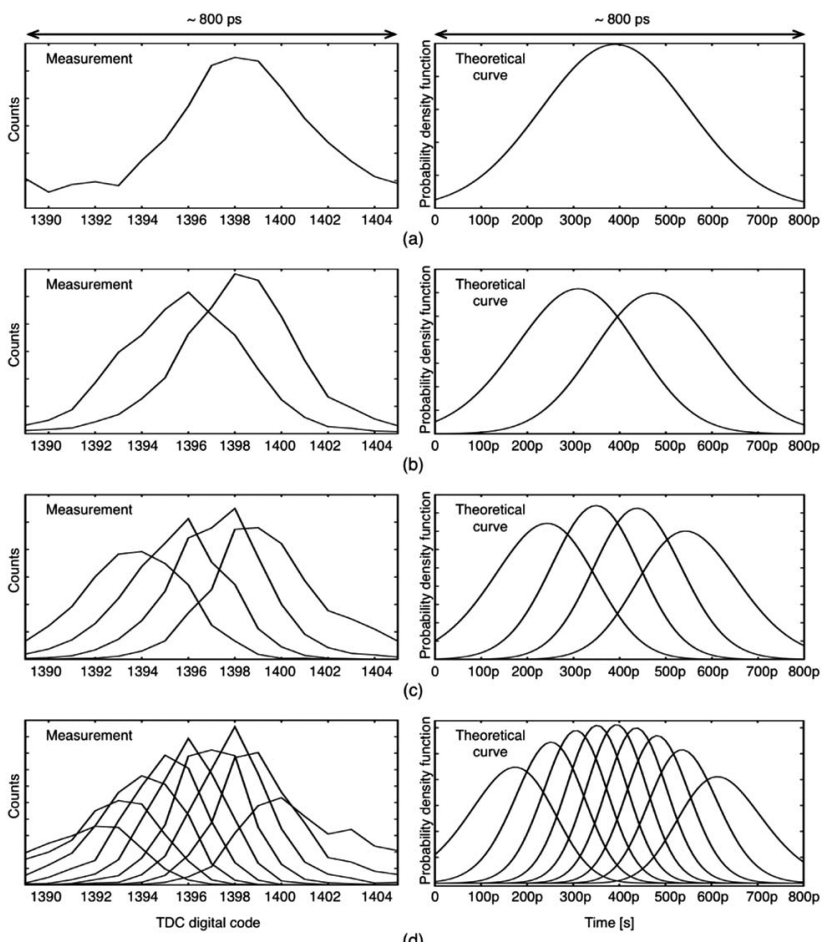

(d)

Fig. 2. Experimental and theoretical p.d.f. curves of each photon at (a) 1 photon level, (b) 2 photon level, (c) 4 photon level, and (d) 9 photon level.

the intrinsic jitter can be estimated to be 70 ps FWHM.

Next, the advantage of utilizing multiple timestamps is further explained. Figure 5 shows the timing resolution at 9 photon level intensity when only one single and multiple timestamps are utilized. When only single photon timestamp is available, the seventh photon has the best timing resolution. However, timing resolution is improved by $50 \%$ and $21 \%$ utilizing multiple timestamps with maximum-likelihood estimation (MLE) by comparing to the first photon and seventh photon timing resolution. In Fig. 6, a comparison between different algorithms is shown to estimate timing information. When only the first photon is utilized, timing resolution cannot be better than any other methods. Even when multiple timestamps

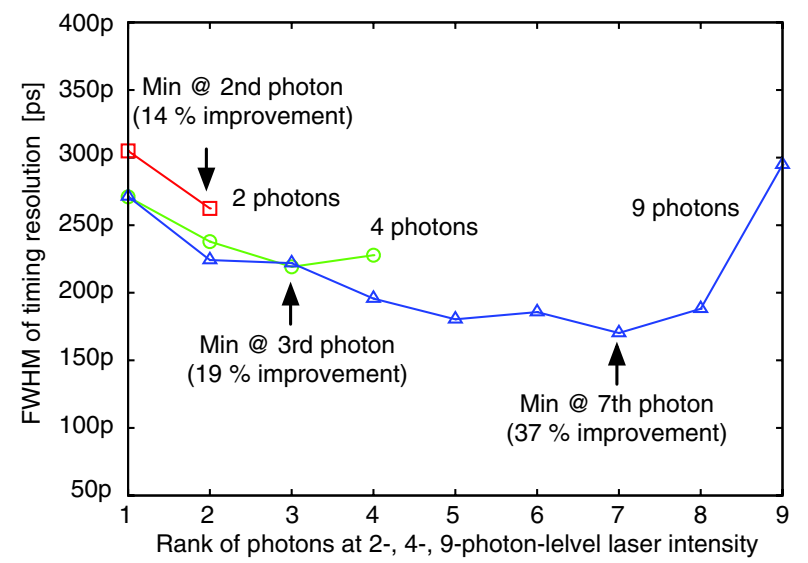

Fig. 3. Timing resolution of each rank of photons at 2, 4, and 9 photon level laser intensity. 


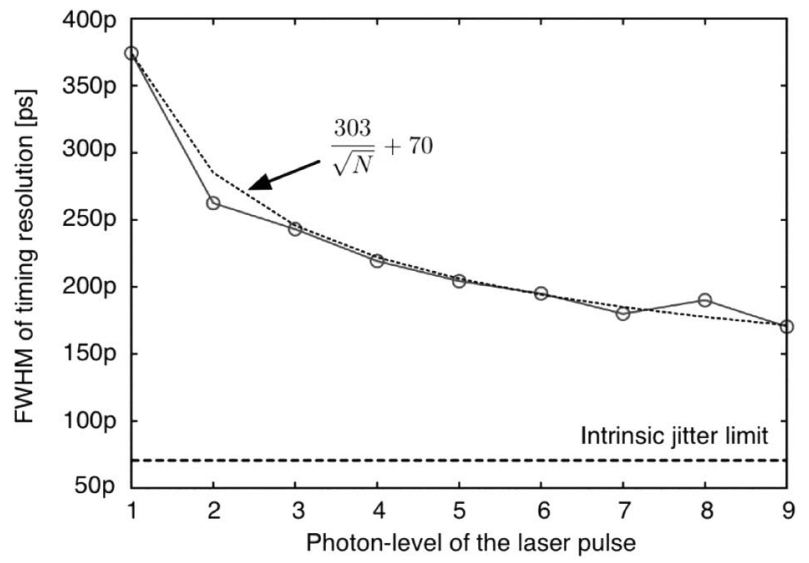

Fig. 4. Timing resolution utilizing single timestamp in each photon-level per laser pulse.

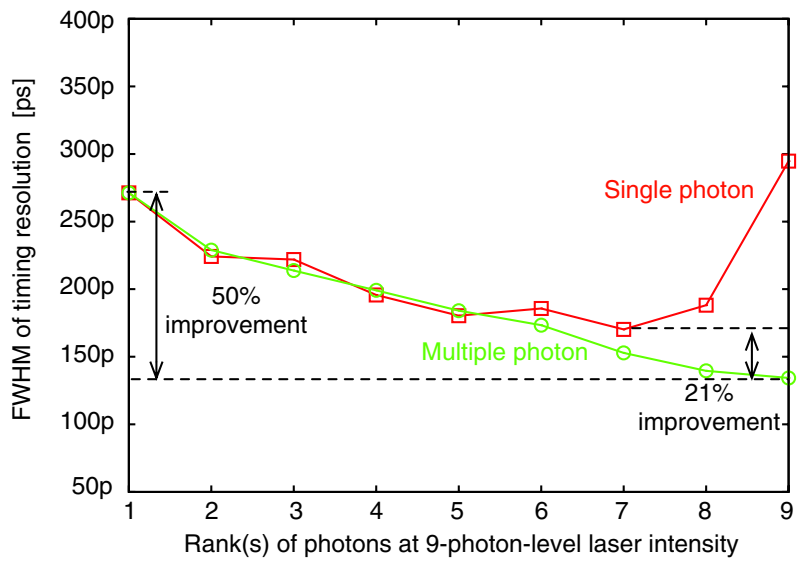

Fig. 5. Timing resolution of each rank of photons and estimated timing resolution utilizing multiple ranks of photons at 9 level photon laser intensity.

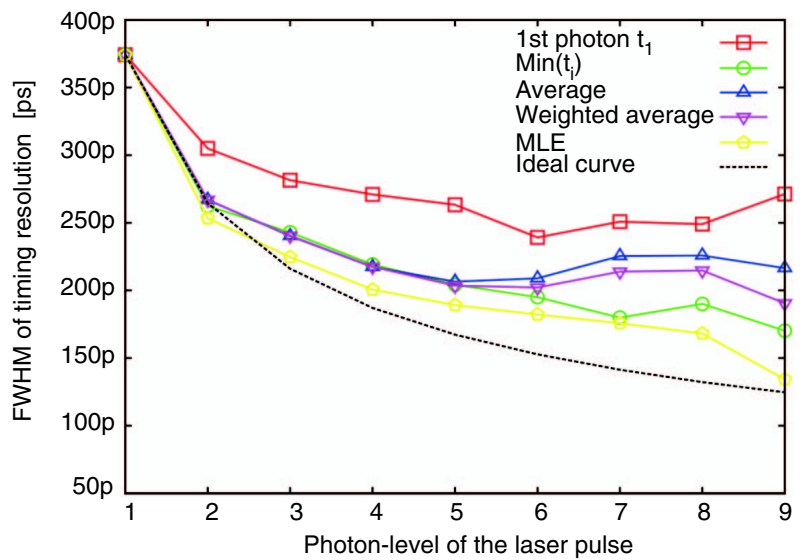

Fig. 6. Comparison of timing resolution with various algorithms.

are available, the suitable algorithm must be chosen. Average and weighed average of multiple timestamps could not be better than choosing the best rank from multiple timestamps. However, MLE could achieve the best performance.

This work has demonstrated the ability of MD-SiPMs to detect multiple photons, and it has verified the advantage of detecting multiple photons assuming incoming photons follow a Gaussian distribution. This work has also shown the benefits of utilizing multiple timestamps to estimate the timing information more accurately than utilizing a single timestamp. The p.d.f. of incoming photons can be arbitrary such as exponential distribution for scintillators in PET as far as each photon is independent and identically distributed random variable, and the timing estimation of events can be accurate by utilizing multiple timestamps. Furthermore, the p.d.f. of incoming photons can be arbitrary, such as an exponential distribution for scintillators in PET. An MD-SiPM can characterize the first several photons's p.d.f. and utilize them for accurately estimating timing information of events.

The research leading to these results has received funding from the European Union Seventh Framework Program under Grant Agreement $\mathrm{n}^{\circ}$ 256984. The authors are also grateful to Xilinx, Inc. for FPGA donations.

\section{References}

1. P. Buzhan, B. Dolgoshein, L. Filatov, A. Ilyin, V. Kantzerov, V. Kaplin, A. Karakash, F. Kayumov, S. Klemin, E. Popova, and S. Smirnov, Nucl. Instrum. Methods Phys. Res. A 504, 48 (2003).

2. A. G. Stewart, V. Saveliev, S. J. Bellis, D. J. Herbert, P. J. Hughes, and J. C. Jackson, IEEE J. Quantum Electron. 44, 157 (2008).

3. N. Zorzi, M. Melchiorri, A. Piazza, C. Piemonte, and A. Tarolli, Nucl. Instrum. Methods Phys. Res. A 636, S208 (2011).

4. M. McClish, P. Dokhale, J. Christian, C. Stapels, E. Johnson, R. Robertson, and K. S. Shah, IEEE Trans. Nucl. Sci. 57, 2280 (2010).

5. MPPC, http://jp.hamamatsu.com.

6. M. Mazzillo, G. Condorelli, D. Sanfilippo, G. Valvo, B. Carbone, A. Piana, G. Fallica, A. Ronzhin, M. Demarteau, S. Los, and E. Ramberg, IEEE Trans. Nucl. Sci. 57, 2273 (2010).

7. T. Frach, G. Prescher, C. Degenhardt, R. de Gruyter, A. Schmitz, and R. Ballizany, in IEEE Nuclear Science Symposium Conference (IEEE, 2009), pp. 1959-1965.

8. C. Veerappan, J. Richardson, R. Walker, D. U. Li, M. W. Fishburn, Y. Maruyama, D. Stoppa, F. Borghetti, M. Gersbach, R. K. Henderson, and E. Charbon, in IEEE International Solid-State Circuit Conference (IEEE, 2011), p. 312.

9. L. Braga, L. Gasparini, L. Grant, R. Henderson, N. Massari, M. Perenzoni, D. Stoppa, and R. Walker, in IEEE International Solid-State Circuit Conference (IEEE, 2013), p. 486.

10. S. Mandai and E. Charbon, in IEEE European Solid-State Circuit Conference (IEEE, 2012), p. 89.

11. S. Mandai and E. Charbon, J. Instrum. 8, 1 (2013).

12. M. W. Fishburn and E. Charbon, IEEE Trans. Nucl. Sci. 57, 2549 (2010).

13. S. Seifert, H. T. van Dam, and D. R. Schaart, Phys. Med. Biol. 57, 1797 (2012).

14. H. T. van Dam, G. Borghi, S. Seifert, and D. R. Schaart, Phys. Med. Biol. 58, 3243 (2013).

15. M. Rohner, K. Siercks, and J. Hinderling, "Single-photon detection for high precision ranging - a trade-off study," in Proceedings of the 1st Range Imaging Research Day, Zurich, Swizerland, 2005, pp. 33-42.

16. S. Seifert, J. H. L. Steenbergen, H. T. van Dam, and D. R. Schaart, J. Instrum. 7, 1 (2013). 